\title{
FUZZY BASED CHIP LEVEL BASED RECEIVER FOR DIRECT SEQUENCE-CODE DIVISION MULTIPLE ACCESS COMMUNICATION SYSTEM
}

\author{
Sharmistha Panda, Sarat Kumar Patra \\ Department of Electronics and Instrumentation Engineering, \\ National Institute of Technology, Rourkela, India-769 008 \\ Tel: +916612463457; Fax: +91661 2472926 \\ e-mail: $\quad$ 20207308, skpatra\}@nitrkl.ac.in
}

\begin{abstract}
This paper investigates the problem of chip level based (CLB) receivers for direct sequence code division multiple access (DS-CDMA) communication system. A radial basis function $(\mathrm{RBF})$ receiver provides the optimum receiver performance for this system. We propose a fuzzy implementation of the RBF receiver. This fuzzy receiver provides considerable computational complexity reduction with respect to RBF receivers. Additionally this fuzzy receivers has the capability to provide exactly the same performance in terms of bit error rate (BER) as the optimum RBF receiver. Extensive simulation studies validate our finding.
\end{abstract}

\section{INTRODUCTION}

The last part of the past mellinium saw the introduction of mobile cellular communication. Ever since the inception there has been a surge in the demand for this technology. Due to limitations in available radio frequency bandwidth, multiple access techniques have been used to provide the users access to the communication channel. First generation communication systems used frequency division multiple access (FDMA) where as second generation systems like IS-54 and GSM used time division multiple access (TDMA) along with FDMA. The third generation systems like IS-95, CDMA2000, universal mobile telecommunication systems (UMTS) used direct sequence code division multiple access (DS-CDMA) techniques. CDMA systems assiogn uncorrelated codes to each mobile users, enabling them to transmit contoinuously over time using the full bandwidth over the complete call duration.DS-CDMA systems use both long and short spreading sequence to provide better capacity [1]

Conventionally RAKE receivers are used in CDMA systems to mitigate the effects of multiple access interference (MAI) in presence of multipath channel effect and additive white Gaussian noise (AWGN). Non linear receivers have been shown to outperform RAKE receivers and minimum

Correspondance may be sent to Sarat Kumar Patra mean square error (MMSE) receivers due to the non-linear decision boundary demanded by the system [2] and the optimum performance is provided by a radial basis function (RBF) receiver.

Few factors have motivated us to use fuzzy systems for CDMA detection. Non-linear receivers using RBF [3], volterra filters, recurrent neural networks [4], multi layer perceptron [5] techniques have been efficiently used for CDMA applications. Most of these techniques have also been used for channel equalization problem. Both channel equalization and CDMA can be considered as pattern classification application where the receiver maps the input signal to finite number of classes. Fuzzy techniques have been efficiently used for channel equalization applications providing computational complexity advantages [6,7], how ever very little has been done in application of fuzzy systems for CDMA receiver design. Work reported herein attempts to make contributions in this line.

This paper is organized as follows. Following this section, DS-CDMA system model is outlined. The next section provides a discussion on adaptive fuzzy filter and its implementation for Multi User Detector (MUD) receiver for DS-CDMA. The performance of the proposed receiver with other standard receivers is discussed next. The last section provides the concluding remarks.

\section{AN OVERVIEW OF DS-CDMS SYSTEM}

System model for DS-CDMA considered here is presented in Figure 1. It shows the downlink scenario, where the mobile unit receives signal $y(k L+n)$ from the base station. The information bits corresponding to the desired user $i$ out of $U$ users are denoted as $x_{i}(k) . x_{i}(k)$ takes the values $+1 /-1$ with equal probability and $k$ denotes the time index of user transmitted symbols. The information bits transmitted by each user are then convolved with each of their mutually orthogonal spreading sequences $C_{i, n}$, where $1 \leq i \leq U$ (number of users active) and $1 \leq n \leq L$ (spreading sequence length). Gold codes, convolutional codes, Pseudo- 


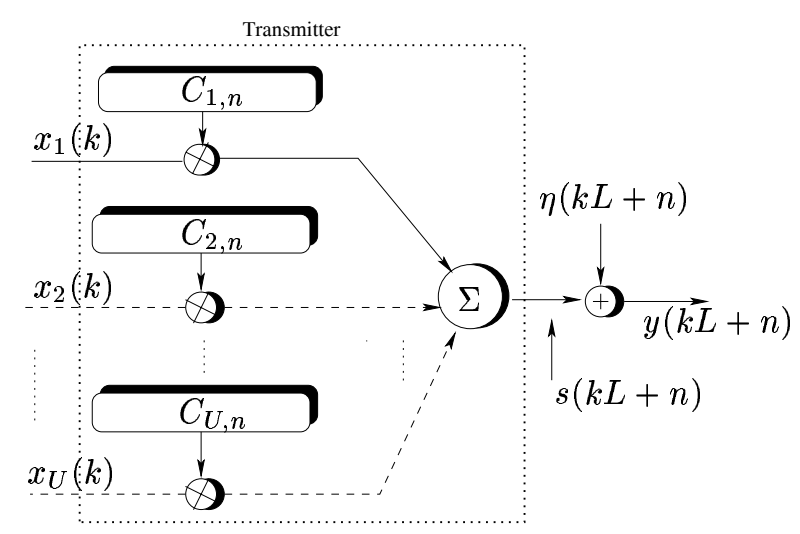

Figure 1: Conventional synchronous DS-CDMA downlink transmitter for $\mathrm{U}$ transmitting users

noise (PN) codes are some of the coding techniques generally used. The spreaded signal from each user are then combined to form

$$
s(k L+n)=\sum_{i=1}^{U} x_{i}(k) C_{i, n}
$$

which is then transmitted through the non-dispersive channel. Channel adds AWGN to the signal. With this the received signal $y(k L+n)$ can be represented as

$$
y(k L+n)=\sum_{i=1}^{U} x_{i}(k) C_{i, n}+\eta(k L+n)
$$

at the point where bit $k$, chip $n$ is received. $\eta(k L+n)$ is the noise component at chip rate. In the AWGN case there is no need to consider $n$ outside the range $1 \leq n \leq L$ as outside this time the signal will contain no information relating to data bit $k$.

The job of the receiver is to estimate the transmitted signal $x_{i}(k)$ of the desired user using the information content in the $y(k L+n)$. As the input signal is processed at chip rate $n$, it is called chip rate based receiver (CLB). The structure of CLB receiver using RBF is shown in Figure 2. The input to the RBF network is given by $y(k L+n)$, and can be represented in vector form $\mathbf{y}(k)=[y(k L+1), y(k L+$ $2), \ldots, y(k L+L)]$ for $1 \leq n \leq L$. The output of the RBF network is given by

$$
t(k)=\sum_{j=1}^{2^{U}} w_{j} \exp \left(\frac{-\left\|\mathbf{y}(k)-\mathbf{c}_{j}\right\|^{2}}{2 \sigma^{2}}\right)
$$

The right side of (3) represents the RBF decision function. The RBF has $2^{U}$ centres of dimension $L, \sigma$ is the centre spread parameter and $w_{j}$ denotes the weight associated with each centre. Each of the components of the $2^{U}$ centres are taken from all possible noise free received scalars. The The RBF output $t(k)$ is passed through a hard limiter to provide

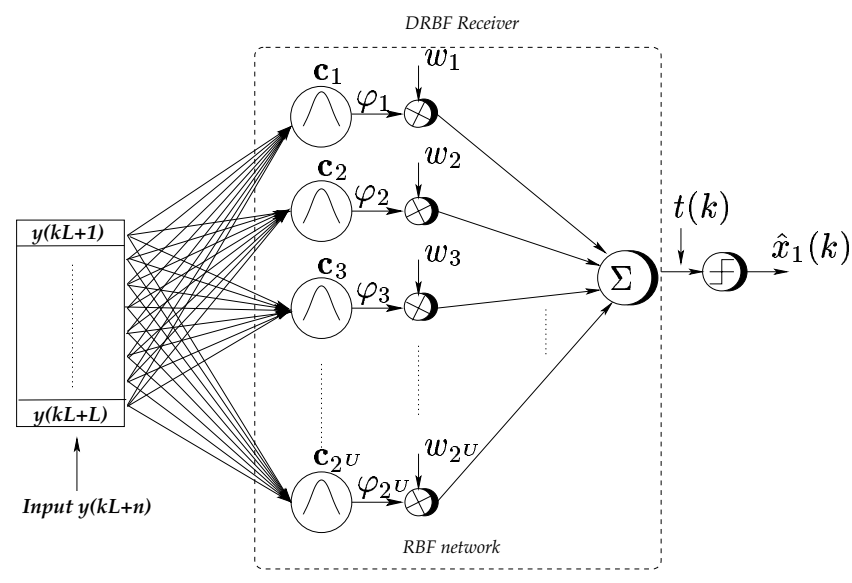

Figure 2: The structure of the CLB RBF receiver

$\hat{x}_{i}(k)$, estimated value of the transmitted symbol of the desired user $x_{i}(k)$. An increment in number of users increases the number of RBF centre by two times. The larger number of centres associated with this Direct Radial Basis Function (DRBF) receivers prompted us to use fuzzy based receivers for this application. This RBF receiver provides the optimal performance for CDMA system [2]. The computational complexity issues associated with these $\mathrm{RBF}$ receivers have been widely investigated and a number of near optimal solutions using neural networks [8], recurrent networks [4] has been investigated in literature.

\section{FUZZY ADAPTIVE FILTER FOR DS-CDMA}

\subsection{Adaptive Fuzzy Filters}

Fuzzy logic system uses linguistic informations to process it's input. The fuzzifier converts the real world crisp input to a fuzzy output described by the membership function. The inference engine provides the relationship between the fuzzy input in terms of membership functions and the fuzzy output of the controller using a set of IF ... THEN ... rules derived from the rule base. The defuzzifier converts the inferences to provide the crisp output. Generally in a fuzzy system the rule base is generated in advance with expert knowledge of the system under consideration. In [7], online learning properties was introduced which provided scope for training the fuzzy system as shown in Figure 3. A typical fuzzy filter is presented in Figure.3.

\subsection{Fuzzy Filter for DS-CDMA chip level based (CLB) Receiver}

In order to propose a fuzzy based DS-CDMA receiver we used a fuzzy filter discussed in subsection 3.1. The close relationship of this filter with RBF was reported by [6]. This technique is used to implement the RBF receiver in 


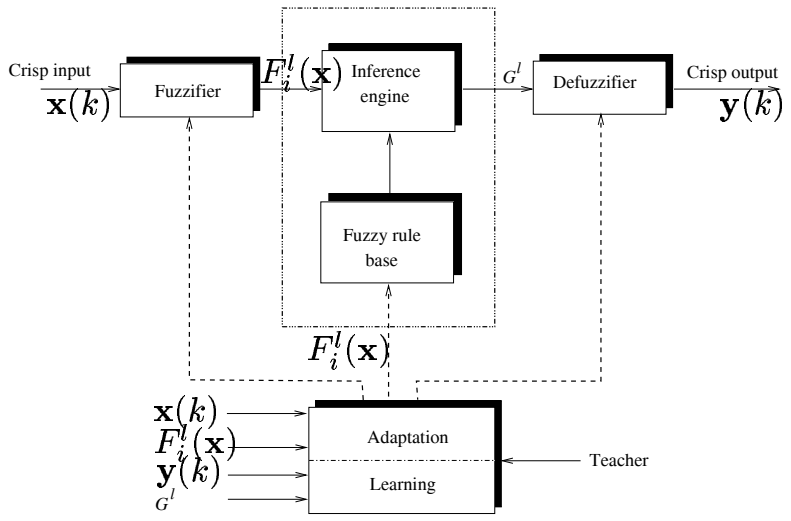

Figure 3: A typical fuzzy logic system

Figure.2. The output of the channel $y(k L+n)$ as shown in Figure 2, provides input to the fuzzy filer. The fuzzy filter consists of a fuzzifier with Gaussian membership function. The centres of the membership function are located at points as shown in the Table 1, which are derived from noise free received signal states for number of users active in the system. This is presented in Table 1. The Table shows all possible noise free received signals for 1 to 8 users active in the system. The fuzzifier convert the crisp received data from the channel into fuzzy variables. The order of the input to the fuzzifier is $L$. With this, there are a total of $(U+1) \times L$ fuzzy inputs corresponding to each set of crisp input of order $L$. The rule base consists of combining one of each membership function from each of the input scalars, hence there are $2^{U}$ rules in the rule base, which are generated by combining all possible fuzzifier output taking one from each input scalar of the input vector. The inference rule used here is product inference. The inference block provides $2^{U}$ outputs generated with product rule. The defuzzification is achieved with COG defuzzifier. It provides a weighted sum of it's input from inference block with it's set of weights. The receiver so designed is presented in Figure 4. This receiver can be considered as an alternative implementation of $\mathrm{RBF}$ receiver. The RBF decision function in (3) discussed in the previous section, for fuzzy implementation can also

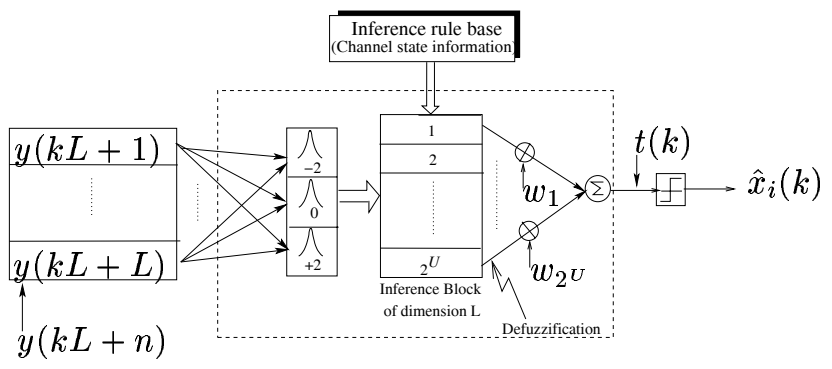

Figure 4: Fuzzy implementation of RBF receiver be represented as

$$
t(k)=\sum_{j=1}^{2^{U}} w_{j}\left\{\prod_{n=1}^{L} \exp \left(\frac{-\left\|y(k L+n)-c_{j, n}\right\|^{2}}{2 \sigma^{2}}\right)\right\}
$$

where $c_{j, n}$ constitute the $n^{t h}$ component of the RBF centre and RBF input. The $\exp ($.$) of Eucleadian distance of$ vectors has been replaced by product of $\exp ($.$) of scalar$ terms of the vector. The system shown in the (4) is represented by the fuzzy system shown in the Figre 4. There are $2^{U}$ rules in the rule base. the product inference block of dimension $L$ provides $2^{U}$ outputs generated with product rule. The defuzzifier provides a weighted sum of it's inputs from inference block with it's set of weights. The weights associated with the defuzzifier can be optimized with adaptive algorithm during the training process with the training data. Figure 4 shows the receiver structure of the receiver when $U=2$ users are active in the system. This proposed receiver (Fuzzy1) can be considered as an alternative implementation of RBF receiver [6]. The Fuzzy1 receiver can be trained with LMS algorithm or algorithms like RLS. The

\begin{tabular}{||c|c||}
\hline$U$ & Possible noise free received scalars \\
\hline 1 & $+1 /-1$ \\
\hline 2 & $+2 / 0 /-2$ \\
\hline 3 & $+3 /+1 /-1 /-3$ \\
\hline 4 & $+4 /+2 / 0 /-2 /-4$ \\
\hline 5 & $+5 /+3+1 /-1 /-3 /-5$ \\
\hline 6 & $+6 /+4 /+2 / 0 /-2 /-4 /-6$ \\
\hline 7 & $+7 /+5 /+3 /+1 /-1 /-3 /-5 /-7$ \\
\hline 8 & $+8 /+6 /+4 /+2 / 0 /-2 /-4 /-6 /-8$ \\
\hline
\end{tabular}

Table 1: Centre locations of the fuzzifier of Fuzzy1 and Fuzzy2 CLB receiver

fuzzy decision function shown in (4) can be further simplified as

$t(k)=\sum_{j=1}^{2^{U}} w_{j}\left\{\min _{n=1}^{L}\left\{\exp \left(\frac{-\left\|y(k L+n)-c_{j, n}\right\|^{2}}{2 \sigma^{2}}\right)\right\}\right\}$

Here the $\prod_{n=1}^{L}$ rule has been replaced by $\min _{n=1}^{L}$ rule which helps further reduction in computational complexity. In this case the input to the fuzzy filter is $y(k L+n)$. This fuzzy filter consists of fuzzifier with Gaussian membership function as shown in Figure 4. The centres of the membership function are located at points as shown in the Table 1, depending upon the number of users simultaneously transmitting in the system. There are $2^{U}$ rules in the rule base. the minimum inference block of dimension $L$ provides $2^{U}$ outputs generated with minimum rule. The defuzzifier provides a weighted sum of it's inputs from minimum inference block with it's set of weights. The weights associated with the de- 
fuzzifier can be optimized with adaptive algorithm like LMS during the training process with the training data. This proposed receiver is termed as Fuzzy 2 receiver with minimum inference rule.

\section{COMPUTATIONAL COMPLEXITY ISSUES}

In this section, we discuss the computational complexity requirements for implementing the fuzzy DS-CDMA CLB receiver. The fuzzy receiver complexity is compared with RBF receiver. The computational complexity for the Fuzzy1, Fuzzy2 and CLB RBF receiver is presented in Table 2. The table presents the general computational complexity associated with the three types of receivers. Computational complexity for receivers with 2 and 7 users are also presented.

\begin{tabular}{||c|l|c|l|l|c||}
\hline$U$ & $\begin{array}{l}\text { Tech- } \\
\text { que }\end{array}$ & $\begin{array}{l}\text { Centres/ } \\
\text { Rule }\end{array}$ & Mul. & $\begin{array}{l}\text { Add/ } \\
\text { Sub/ } \\
\text { Comp }\end{array}$ & exp(.) \\
\hline \multirow{3}{*}{2} & RBF & 4 & 32 & 28 & 4 \\
\cline { 2 - 6 } & Fuzzy1 & 4 & 49 & 25 & 21 \\
\cline { 2 - 6 } & Fuzzy2 & 4 & 25 & 49 & 21 \\
\hline \multirow{3}{*}{7} & RBF & 128 & 1024 & 896 & 128 \\
\cline { 2 - 6 } & Fuzzy1 & 128 & 952 & 184 & 56 \\
\cline { 2 - 6 } & Fuzzy2 & 128 & 184 & 952 & 56 \\
\hline
\end{tabular}

Table 2: Computational complexity for CLB receivers using RBF, Fuzzy1 and Fuzzy2

From the table it is seen that, when 2 users are active, the $\mathrm{RBF}$ receiver will have $2^{U}=4$ centres each with a dimensionality of $L=7$. The Fuzzy receivers will have $(U+1)$ membership function and $2^{U}=4$ product and minimum inference rules respectively. When the number of users increased to 7 , the number of RBF centres increases to 128 same as the number of inference rules in the Fuzzy CLB receivers. From the table it can be seen that, the fuzzy based CLB receivers provide considerable computational complexity reduction in terms of multiplication, addition and $\exp ($.$) calculations. Additionally the computational com-$ plexity advantages achieved with the fuzzy receiver increases with increase in number of active users in the system.

\section{SIMULATION RESULTS}

In order to validate the proposed fuzzy CLB receivers for DS-CDMA applications, extensive simulation studies were conducted. The results obtained were compared with the CLB receiver using RBF network and simple linear receiver like Matched Filter (MF). All the simulation studies were conducted on a $P-I V @ 1.9 \mathrm{GHz} \mathrm{PC}$ with $512 \mathrm{MB}$ of RAM, Redhat 9.0 operating system. GNU C++ compiler was used to test the simulations. During the training period the receiver parameters were optimized/ trained with 1000 ran- dom samples and the parameters so obtained were averaged over 50 experiments. The parameters of the receiver were fixed after the training phase. The receiver weights were trained using LMS algorithm.

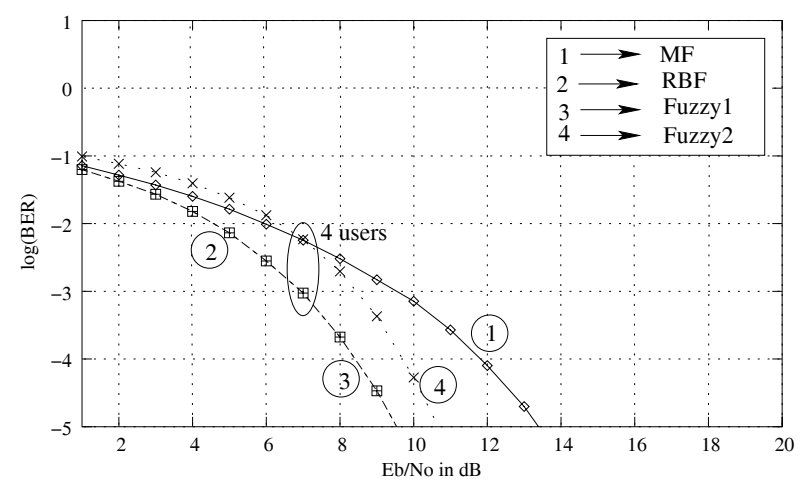

Figure 5: BER performance for varying $E_{b} / N_{o}$ with 4 users being active in the system

Bit error rate (BER) was considered as the performance index. Monte Carlo simulation were conducted to estimate the BER performance of the fuzzy CLB receivers and the performance was compared with CLB RBF and linear MF receivers. In all the experiments randomly generated $+1 /-$ 1 samples were transmitted for each user. 7 bit Gold codes were used for spreading the signal of each user. This restricted the maximum permissible user's in the system to 7 . After spreading, the sequences were added and transmitted through the non-dispersive channel. The channel corrupted the transmitted signal with AWGN. The channel output was fed to the various receiver structures. A total of $10^{7}$ bits were transmitted by each user and a minimum of 1000 errors were recorded. The tests were conducted for different levels of $E_{b} / N_{o}$. Where $E_{b}$ is the energy per bit of the tranemitted signal and $N_{0}$ is the two sided noise spectral density. Additionally tests were also conducted by varying number of active users in the system for fixed value of $E_{b} / N_{o}$.

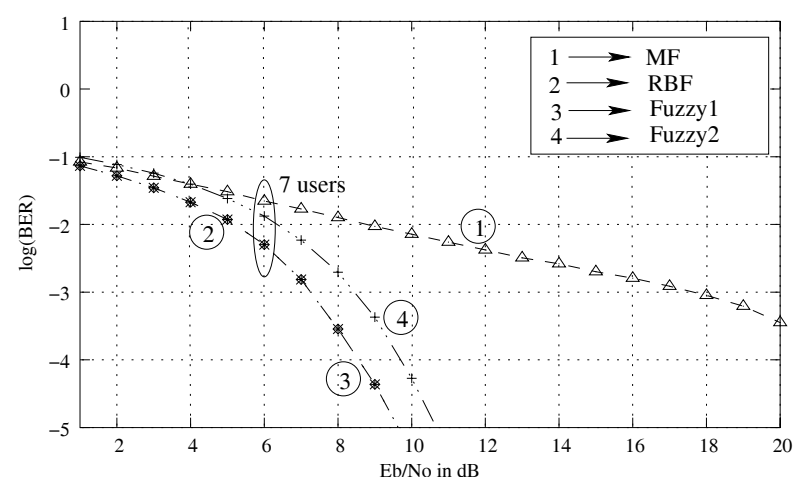

Figure 6: BER performance for varying $E_{b} / N_{o}$ with 7 users being active in the system 
BER performance of the four type of receivers with 4 and 7 users active in the system is shown in the Figure 5 and Figure 6 respectively for various values of $E_{b} / N_{o}$ in the channel. From Figure 5 it can be seen that the performance degradation of the MF at a BER of $10^{-5}$ is about $4 \mathrm{~dB}$ as compared to the RBF/ Fuzzy1 CLB receiver and about $2.5 \mathrm{~dB}$ as compared to the Fuzzy2 CLB receiver. Similarly from the Figure 6 it is seen that performance degradation of the MF at a BER of $10^{-3}$ is about $10 \mathrm{~dB}$ as compared to the $\mathrm{RBF} /$ Fuzzy1 CLB receiver and about $7.5 \mathrm{~dB}$ as compared to the Fuzzy2 CLB receiver. The performance degradation of the MF is high as active users $U$ increases in the system as compared to RBF/ Fuzzy1/ Fuzzy2 CLB receiver.

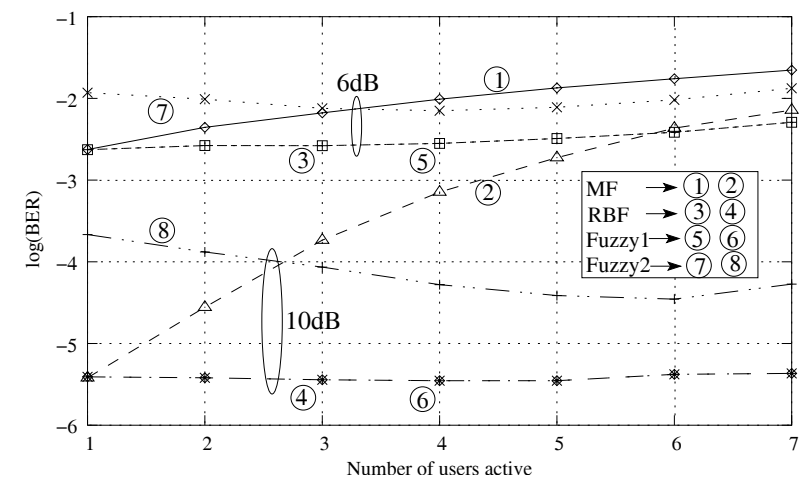

Figure 7: Performance of chip-level receivers in AWGN, 7-chip spreading sequence, with varying number of users active in the system

Subsequently the BER performance of the receivers were studied for $E_{b} / N_{o}$ values of $6 \mathrm{~dB}$ and $10 \mathrm{~dB}$ at the channel output against change in loading in form of number of users being active in the system. The results were plotted in Figure 7. From this it can be seen that Fuzzy1 receiver performs exactly same as the optimal DRBF receiver. The performance of the proposed Fuzzy 2 receiver is in between $\mathrm{MF}$ and the optimal RBF receiver for all loading conditions. Here the number of active users are varied from 1 to 7. From the graph it is seen that for a fixed value of BER when $E_{b} / N_{o}$ at the channel output improves, more number of users can be accommodated in the system. The simulation studies show that the proposed Fuzzy1 receiver is an implementation of optimal RBF receiver and provides the same performance for a reduced computational complexity, where as the proposed Fuzzy 2 receiver provides performance in between MF and RBF receiver with reduced computational complexity compared to RBF receiver. This provides a performance tradeoff for complexity.

\section{CONCLUSION}

In this paper RBF based CLB receiver has been implemented with fuzzy system. The fuzzy receivers proposed uses Gaus- sian membership function, product/ minimum inference and centre of gravity (COG) defuzzifier. The Fuzzy1 receiver with product inference provides computational complexity reduction over the optimal $\mathrm{RBF}$ receiver and it provides a performance exactly same as the RBF receiver. The proposed Fuzzy 2 receiver with minimum inference provides further reduction in computational complexity over the optimal DRBF receiver outperforms the conventional MF but the performance is poor as compared to the optimal DRBF.

\section{ACKNOWLEDGEMENT}

The work conducted here was facilitated by Ministry of $\mathrm{Hu}-$ man Resource Development (MHRD), Govt of India, in the form of R\& D project, titled Development of Fuzzy Receivers for DS-CDMA System.

\section{REFERENCES}

[1] D. Shiung and Jin-Fu Chang, "Enhancing the Capacity of DS-CDMA System Using Hybrid Spreading Sequences," IEEE Transactions on Communications, vol. 52, pp. 372-375, March 2004.

[2] B. Mulgrew, "Applying Radial Basis Functions," IEEE Signal Processing Magazine, vol. 13, pp. 50-65, March 1996.

[3] D. G. M. Cruickshank, "Radial Basis Function Receivers for DS-CDMA," IEE Electronics Letter, vol. 32, pp. 188-190, February 1996.

[4] M. Benson and R. A. Carrasco, "Application of a Recurrent Neural Network to Space Diversity in SDMA and CDMA Mobile Communication Systems," Neural Comput \& Applic, Springer-Verlang London Limited, no. 10, pp. 136-147, 2001.

[5] U. Mitra and H. V. Poor, "Neural Network Techniques for Adaptive Multiuser Demodulation," IEEE Transactions on Selected Areas in Communications, vol. 12, pp. 1460-1470, December 1994.

[6] S. K. Patra and B. Mulgrew, "Fuzzy Techniques for Adaptive Nonlinear Equalization," Signal Processing, vol. 80, pp. 985-1000, Jan 2000.

[7] L.-X. Wang, Adaptive Fuzzy Systems and Control : Design and Stability Analysis. Englewood Cliffs, N.J., USA: Prentice Hall, 1994.

[8] M. G. Shayesteh and Hamidreza Amindavar, "Neural Networks for Multiuser Detection of Signals in DS/CDMA Systems," Neural Comput \& Applic, Springer-Verlang London Limited, no. 11, pp. 178-190, 2003. 